Full length article

\title{
Magnetic responsive materials modulate the inflammatory profile of IL- $1 \beta$ conditioned tendon cells
}

\author{
A. Vinhas ${ }^{a, b}$, M.T. Rodrigues ${ }^{a, b}$, A.I. Gonçalves ${ }^{a, b}$, R.L. Reis ${ }^{a, b}$, M.E. Gomes ${ }^{a, b, *}$ \\ a 3B's Research Group, I3Bs - Research Institute on Biomaterials, Biodegradables and Biomimetics, University of Minho, Headquarters of the European \\ Institute of Excellence on Tissue Engineering and Regenerative Medicine, AvePark, Parque de Ciência e Tecnologia, Zona Industrial da Gandra, $4805-017$ \\ Barco, Guimarães, Portugal \\ b ICVS/3B's - PT Government Associate Laboratory, Braga, Guimarães, Portugal
}

\section{A R T I C L E I N F O}

\section{Article history:}

Received 28 May 2020

Revised 10 September 2020

Accepted 15 September 2020

Available online $\mathrm{xxx}$

\section{Keywords:}

Magnetic responsive membranes

Nanoparticles

Magnetic platforms

Tendon cells

Inflammatory environmental cues

\begin{abstract}
A B S T R A C T
Tendinopathies represent half of all musculoskeletal injuries worldwide. Inflammatory events contribute to both tendon healing and to tendinopathy conditions but the cellular triggers leading to one or the other are unknown. In previous studies, we showed that magnetic field actuation modulates human tendon cells (hTDCs) behavior in pro-inflammatory environments, and that magnetic responsive membranes could positively influence inflammation responses in a rat ectopic model.
\end{abstract}

Herein, we propose to investigate the potential synergistic action of the magnetic responsive membranes, made of a polymer blend of starch with polycaprolactone incorporating magnetic nanoparticles (magSPCL), and the actuation of pulsed electromagnetic field (PEMF): $5 \mathrm{~Hz}, 4 \mathrm{mT}$ of intensity and $50 \%$ of duty cycle, in IL-1 $\beta$-treated-hTDCs, and in the immunomodulatory response of macrophages.

It was found that the expression of pro-inflammatory (TNF $\alpha, I L-6, I L-8, C O X-2)$ and ECM remodeling (MMP-1,-2,-3) markers tend to decrease in cells cultured onto magSPCL membranes under PEMF, while the expression of TIMP-1 and anti-inflammatory genes $(I L-4, I L-10)$ increases. Also, CD16 ${ }^{++}$and CD206 ${ }^{+}$ macrophages were only found on magSPCL membranes with PEMF application.

Magnetic responsive membranes show a modulatory effect on the inflammatory profile of hTDCs favoring anti-inflammatory cues which is also supported by the anti-inflammatory/repair markers expressed in macrophages. These results suggest that magnetic responsive magSPCL membranes can contribute for inflammation resolution acting on both resident cell populations and inflammatory cells, and thus significantly contribute to tendon regenerative strategies.

\section{Statement of significance}

Magnetically-assisted strategies have received great attention in recent years to remotely trigger and guide cell responses. Inflammation plays a key role in tendon healing but persistent pro-inflammatory molecules can contribute to tendon disorders, and therefore provide a therapeutic target for advanced treatments.

We have previously reported that magnetic fields modulate the response of human tendon cells (hTDCs) conditioned to pro-inflammatory environments (IL- $1 \beta$-treated-hTDCs), and that magnetic responsive membranes positively influence immune responses. In the present work, we combined pulsed electromagnetic field (PEMF) and magnetic responsive membranes to guide the inflammatory profile of IL- $1 \beta$ treated-hTDCs and of macrophages. The results showed that the synergistic action of PEMF and magnetic membranes supports the applicability of magnetically actuated systems to regulate inflammatory events and stimulate tendon regeneration.

(c) 2020 Acta Materialia Inc. Published by Elsevier Ltd. All rights reserved.

\footnotetext{
* Corresponding author.

E-mail address: megomes@i3bs.uminho.pt (M.E. Gomes).
}

\section{Introduction}

Tendinopathy defines a multifactorial spectrum of multiple disorders often resulting from overuse activities and/or excessive me- 
chanical loading [1,2]. Tendinopathies are a significantly clinical problem, accounting for almost $50 \%$ of all musculoskeletal afflictions worldwide [3,4]. Clinical diagnosis of tendinopathy relies on gradual onset of activity-related pain, decreased function and sometimes localized swelling. Current therapies target pain relief and consist of oral and locally administered anti-inflammatory drugs. Severe injury often requires surgery but in either case, the treatment is not effective, as it fails to reinstate tendon function following injury $[1,2,5]$. Inflammation is a necessary process for healing, but chronic inflammation has been related to several tendinopathies in tendons with different functions and anatomical locations [5].

Macrophages modulate inflammatory cascades switching their own phenotype to environmental stimuli. The balance between pro-inflammatory and pro-repair macrophage populations (M1 and $\mathrm{M} 2$, respectively) dictates inflammation regulation and resolution, being critical for improved healing [6]. Although recent studies show that macrophages are present in injured tendons, their influence on tendon resident cells and in tendon repair is unclear $[7,8]$. Thus, studies aimed at finding appropriate tools for modulating inflammation and prospecting tissue regeneration, open positive perspectives for new therapeutic approaches to treat tendon disorders.

Pulsed electromagnetic field (PEMF) has been clinically applied to manage pain and delayed wound healing via remote actuation and minimally invasive approaches $[9,10]$. In a previous work, we showed that a particular set of PEMF parameters modulated the pro-inflammatory profile of IL- $1 \beta$-conditioned tendon cells influencing intracellular communication associated to MAPK/Erk(1/2) pathway and connexin-43 [11]. Other studies also showed that the length of exposure, the field intensity and the number of PEMF treatments differently affect tendon cell proliferation, gene expression and the kinetics released of pro- and anti-inflammatory cytokines [12].Furthermore, the impact of PEMF was reported to enhance stem cells proliferation [13] and tenogenic differentiation $[14,15]$, likely providing mechanical cues to the cells $[16,17]$, strengthening the potential of magnetic stimuli to remotely trigger and modulate cell responses.

The combination of magnetic actuation with magnetic responsive biomaterials has emerged as a platform enabling a precise action over biological processes in tissue engineering and regenerative medicine (TERM) strategies [18,19], foreseeing sophisticated, remotely controlled systems with higher therapeutic potential. Our group has recently demonstrated that magnetic responsive fibrous scaffolds can improve in vitro tenogenesis of mesenchymal stem cells through mechano-sensing mechanisms [20], and that the combination of magnetic responsive biomaterials and magnetic stimuli favors immunomodulatory responses that may contribute to tendon healing and to functional recovery [21,22]. Additionally, strategies resourcing to magnetic responsive constructs are envisioned to potentiate more effective and real time monitoring treatments $[18,22-25]$.

Consequently, we hypothesized that magnetic responsive biomaterials exposed to an external magnetic field could have a synergistic action in modulating the inflammatory profile of tendon cells after IL- $1 \beta$ stimulation.

We and others have observed that IL- $\beta$ stimulates proinflammatory cues in tendon cells and could be used as an in vitro model in tendon inflammation studies $[7,26,27]$. Thus, tendon cells previously treated with IL- $1 \beta$ were cultured on magnetic responsive membranes made of a polymeric blend of poly- $\varepsilon$-caprolactone and starch (SPCL) incorporating iron oxide nanoparticles (magSPCL) and exposed to a magnetic field, whose intensity, frequency and duty cycle were previously optimized [11]. These membranes were previous studied, showing promising outcomes in modulating in- flammation and the growth of fibrous tissue in an ectopic rodent model [22].

The phenotype and inflammatory profile of tendon cells was then investigated and compared to non-PEMF conditions. We further hypothesized that PEMF actuated magSPCL membranes could also influence macrophage populations in pro-inflammatory environments. Thus, human macrophages were cultured on magSPCL membranes and markers associated to pro-inflammatory (M1) and repair (M2) phenotypes were investigated to provide on the immuno-modulatory effect of the PEMF and magSPCL membranes on inflammatory cells envisioning complementary and improved oriented strategies headed for tendon repair and regeneration.

\section{Materials and methods}

\subsection{MagSPCL membranes preparation}

Membranes made of a 30:70 blend of starch and polycaprolactone (SPCL, Novamont, Novara, Italy) were produced using the solvent casting method as reported before [22,28]. Briefly, SPCL granules were dissolved in chloroform 20\% (w/v) (VWR, Darmstadt, Germany). Then, the polymeric solution was doped with commercial iron oxide magnetic nanoparticles $\left(\mathrm{Fe}_{3} \mathrm{O}_{4}\right.$, magnetite, MNPs) (Micromod, 45-00-252, Rostock, Germany). These MNPs form monodisperse magnetic aggregates $(\varphi 250 \mathrm{~nm}$ ), exhibit a plain surface and a polydispersity index $<0.2$. MNPs are stable in aqueous buffers with $\mathrm{pH}>4$, and present a magnetization value of $46 \mathrm{emu} / \mathrm{g}$ iron $(H=1000 \mathrm{Oe})$, a saturation magnetization $>71$ emu/g iron $(H>10.000 \mathrm{Oe})$ and a coercive field Hc of $0.481 \mathrm{Ka} / \mathrm{m}$. MNPs were thoroughly dispersed in the SPCL/chloroform solution by sonication (DT100H SONOREX; VWR), which provided energy to agitate and distribute particles in the membrane, and occasional orbitation during $30 \mathrm{~min}$ at a temperature of $20{ }^{\circ} \mathrm{C}-25^{\circ} \mathrm{C}$, before casted in glass petri dishes. After detaching, membranes were washed with ultrapure water and dried. The magnetic formulation was created with SPCL/1.8\% MNPs (w/w). The casted membranes, previously shown to display an average thickness of $0.19 \pm 0.02 \mathrm{~mm}$ [23], were cut into $1 \mathrm{~cm}^{2}$ discs using a hollow punch and sterilized by exposure to an UV lamp for $30 \mathrm{~min}$ before the cell culture studies.

\subsection{Cells isolation and expansion}

Human tendon derived cells (hTDCs) were isolated from surplus tissue samples collected from patients undergoing orthopedic reconstructive surgeries under protocols previously established with Hospital da Prelada (Porto, Portugal) and with informed consent of the patients. The content of the written informed consent and related procedures were reviewed and approved by the Hospital Ethics Committee (P.I. N. ${ }^{\circ} 005 / 2019$ ). The healthy nature of tendons was confirmed by the orthopedic surgeon at the surgical theater upon harvesting of tendon tissues.

Human tendon derived cells were isolated and cultured as described before [29-31]. Briefly, tissue explants were immersed in a sterile solution of phosphate buffer saline (PBS, Sigma-Aldrich, Saint Louis, MO, USA) with $2 \%$ antibiotic/antimicotic solution (A/A, Alfagene, Life Technologies Limited, Paisley, UK). Then, tissue samples were minced and digested in an enzymatic solution of $0.1 \%$ (v/v) of collagenase (Sigma-Aldrich, C6885, Saint Louis, MO, USA) with $2 \mathrm{M} \mathrm{CaCl}_{2}$ (VWR, Darmstadt, Germany) (1:1000) and 1\% bovine serum albumin (BSA, Sigma-Aldrich, Saint Louis, MO, USA) for $1 \mathrm{~h}$ at $37{ }^{\circ} \mathrm{C}$ under agitation. After incubation, digested samples were filtered using a cell strainer $(100 \mu \mathrm{m}$, Enzifarma, 352,360) adapted for $50 \mathrm{ml}$ conical tubes (Falcon), centrifuged three times at $290 \mathrm{~g}$ for $5 \mathrm{~min}$, and the supernatant discarded. hTDCs were ex- 
panded in basic culture medium composed of $\alpha$-MEM (A-MEM, Invitrogen, Life Technologies Limited, Paisley, UK) supplemented with 10\% FBS (Alfagene, Life Technologies Limited, Paisley, UK) and 1\% A/A solution (Alfagene, Life Technologies Limited, Paisley, UK).

\subsection{Human peripheral blood mononuclear cells isolation}

Human peripheral blood mononuclear cells (PBMCs) were obtained from blood donations of healthy adult volunteers and collected into a heparin containing tubes to avoid coagulation. Human macrophages were obtained from buffy coat fractions separated form blood donation units of healthy volunteers under an cooperation agreement established with the Portuguese Institute of Blood and Transplantation (IPST, Instituto Português do Sangue e Transplantação, Portugal). Then, the samples were diluted in 2-4 vol of PBS containing BSA. PBMCs were isolated from buffy coats using a density gradient cell separation medium, Histopaque (SigmaAldrich, Saint Louis, MO, USA), after centrifugation at $800 \mathrm{~g}$ for $30 \mathrm{~min}$ at room temperature. The mononuclear cell layer, comprising lymphocytes and monocytes, was collected into a tube already containing a PBS/BSA solution and centrifuged at $350 \mathrm{~g}$ for $10 \mathrm{~min}$. The pellet was resuspended in PBS/BSA and centrifuged in 3 cycles at $4{ }^{\circ} \mathrm{C}$ at $350 \mathrm{~g}$ for $5 \mathrm{~min}$. Then, cells were positively immunomagnetically sorted using human CD14+ beads (microBeads, Miltenyi Biotec, Bergish Gladbach, Germany) and cultured in RPMI1640 medium (Sigma-Aldrich, Saint Louis, MO, USA) supplemented with $50 \mathrm{ng} / \mathrm{mL}$ of macrophage colony-stimulated factor (M-CSF, Peprotech, Rocky Hill, NJ, USA) for 6 days to differentiate into M0type macrophages.

\subsection{Cell culture on magSPCL membranes (constructs) under magnetic stimulation}

Human TDCs cells were seeded at a density of $1 \times 10^{5}$ cells per membrane in $\alpha$-MEM medium (A-MEM, Invitrogen, Life Technologies Limited, Paisley, UK). hTDCs were treated for $24 \mathrm{~h}$ with exogenous supplementation of IL- $1 \beta$ ( $1 \mathrm{ng} / \mathrm{mL}$, Alfagene, Life Technologies Limited, Paisley, UK) after cell attachment and $24 \mathrm{~h}$ after seeding of tendon cells. Then, cells were cultured for 1,3 and 7 days, as previously established [11]. Similarly, human macrophages were seeded at $2 \times 10^{5}$ cells per membrane and cultured in RPMI medium (Sigma-Aldrich, Saint Louis, MO, USA). Both experiments were conducted under both static (control, non-PEMF) and magnetic stimulation. A magneto therapy device (Magnum XL Pro, Globus, Globus Corporation, Italy) was used to generate a controlled magnetic field with a frequency of $5 \mathrm{~Hz}, 4 \mathrm{mT}$ of intensity and $50 \%$ of duty cycle in accordance to previous studies [11]. Cells were magnetically stimulated for a single $1 \mathrm{~h}$ period.

The rationale behind the selection of these PEMF parameters and the exposure time was to stimulate the cells just enough time to elicit a biological response, in this case, to modulate tendon cells inflammatory profile.

The stimulus provided was selected from a set of therapeutic programmes used in health-rehab medical treatments, pre-defined in the magnetotherapy device.

Cell laden membranes were placed and cultured between two coils (solenoids). The magnetic field was generated by the electric current that goes through a coil (solenoid) with a vertical action between the coils. Afterwards, the system was moved inside an incubator, where the temperature was maintained at $37{ }^{\circ} \mathrm{C}$ in humidified 5\% $\mathrm{CO}_{2}$ atmosphere during the PEMF stimulation. The PEMF ( $5 \mathrm{~Hz}, 4 \mathrm{mT}$ and $50 \%$ of duty cycle) was applied directly over cell culture plates inside the incubator.

Two experimental controls were considered: i) hTDCs seeded on magSPCL membranes cultured in non- PEMF stimulating (static) conditions to investigate PEMF vs non-PEMF conditions, and ii) hTDCs seeded on magSPCL membranes in non-PEMF stimulating conditions without IL- $1 \beta$ treatment to assess tendon cell responses in non-stimulated culture conditions.

Cells-membrane constructs were further investigated for cell viability, proliferation and mRNA transcriptional levels of inflammatory and tendon-associated markers.

\subsection{Morphological characterization of magnetic responsive cell laden membranes}

After 1,3 and 7 days in culture, cells laden onto magnetic responsive membranes were washed with phosphate buffer saline (PBS) and fixed in $4 \%$ formaldehyde for $30 \mathrm{~min}$ at room temperature. Subsequently, samples were dehydrated using an ascending solution of ethanol (from 30 to $100 \%$ ), followed by a $5 \mathrm{~min}$ immersion in hexamethyldisiloxane (HMSO, Sigma-Aldrich, Saint Louis, MO, USA). Next, samples were air-dried overnight and sputter coated (30 s at $20 \mathrm{~mA}$, Cressington, C5219, Model 108A) with gold. Images of the constructs were acquired using a scanning electron microscope (SEM, JEOL, Japan).

\subsection{Human TDCs (hTDCs) characterization}

\subsubsection{Metabolic activity and cell proliferation}

The metabolic activity and proliferation of hTDCs were evaluated by MTS assay (CellTiter 96® AQueous One Solution, Promega) and Quant-It PicoGreen dsDNA assay Kit (Thermo Fisher Scientific), respectively. For the MTS assay, cell laden membranes were washed with PBS and incubated with a mixture of serum-free culture medium without phenol red and MTS solution (5:1 ratio) for $3 \mathrm{~h}$ at $37{ }^{\circ} \mathrm{C}$ and $5 \% \mathrm{CO}_{2}$ atmosphere, protected from light. After this period, the supernatant was transferred to a 96-well plate and the absorbance read at $490 \mathrm{~nm}$ (Synergy ${ }^{\mathrm{TM}} \mathrm{HT}$, BIO-TEK Instruments, Winooski, Vermont). Samples were read in triplicates and a blank sample (no cells) was assessed as control of the assay.

For the dsDNA quantification assay, samples were washed with PBS and then transferred to a microtube with $1 \mathrm{ml}$ of sterile ultrapure water and kept at $-80{ }^{\circ} \mathrm{C}$ until analysis. Samples were then thawed, sonicated and analyzed according to the manufacturer's instructions, at an excitation/emission wavelength of 485/528 nm (Synergy ${ }^{\mathrm{TM}} \mathrm{HT}$, BIO-TEK Instruments, Winooski, Vermont). Samples and standards were made in triplicate.

\subsubsection{Immunofluorescence for tendon related-markers}

Cell laden membranes cultured for 1, 3 and 7 days were washed three times with PBS, before and after fixation with $10 \%(\mathrm{v} / \mathrm{v})$ neutral buffered formalin (Thermo Fisher Scientific) overnight and kept in PBS at $4{ }^{\circ} \mathrm{C}$ until usage. To detect tendon ECM proteins, antibodies against collagen type I (Rabbit polyclonal AntiCollagen I, ab9039, 1:500, Abcam, Cambridge, UK) and Tenomodulin (Rabbit anti-human, ab81328, 1:100, Abcam, Cambridge, UK) were used. The immunolocation of Scleraxis (Rabbit anti-human, ab58655, 1:100, Abcam, Cambridge, UK), NFkB p65 (Rabbit antihuman, F0514, 1:200, Santa Cruz Biotechnology, Heidelberg, Germany), alpha smooth muscle Actin (Rabbit anti-human, ab32575, 1:200, Abcam, Cambridge, UK) and Vinculin (Mouse anti-human, V9131, 1:300, Sigma-Aldrich, Saint Louis, MO, USA) were also assessed.

The cells cultured on the magSPCL membranes were firstly permeabilized with $0.025 \%$ (v/v) Triton X-100 (Sigma-Aldrich, Saint Louis, MO, USA) prepared in PBS for $10 \mathrm{~min}$. Then, constructs were washed three times with PBS, blocked with Normal Horse Serum (RTU Vectastain Kit, PK-7200, Vector, Burlingame, California USA) and incubated overnight with the antibodies diluted in antibody diluent with background reducing components (Dako, Santa Clara, 
CA, USA) at $4{ }^{\circ} \mathrm{C}$. Afterwards, samples were rinsed in PBS, following by inactivation of endogenous peroxidase activity with hydrogen peroxide solution $(0.3 \% \mathrm{w} / \mathrm{v}$, Panreac). The samples were incubated for $1 \mathrm{~h}$ at room temperature with fluorescent secondary antibodies Alexa fluor 488 donkey anti-rabbit (2072687, 1:1000, Alfagene, Life Technologies Limited, Paisley, UK) or Alexa fluor 488 rabbit antimouse (2005937, 1:100, Alfagene, Life Technologies Limited, Paisley, UK), according to the host species of the primary antibodies. Constructs were rinsed in PBS and stained with 4,6-Diamidino-2phenyindole, dilactate (DAPI, $5 \mathrm{mg} / \mathrm{ml}$, D9564, Sigma-Aldrich, Saint Louis, MO, USA) for 10 min. Finally, samples were incubated with a Phalloidin-Tetra- methylrhodamine B isothiocyanate (Phalloidin, P1951, 1:200, Sigma-Aldrich, Saint Louis, MO, USA) solution, which was prepared according to manufacturer's instructions.

Surface specific markers for macrophage phenotype were assessed using conjugated antibodies: CD14 PE (Anti-human, B244291, Biolegend), CD16 APC (anti-human, B2128291, Biolegend), CD45 FITC (anti-human, 555482, BD Biosciences), CD68 APC (antihuman, B178818, Biolegend), CD169 (alexa fluor 647 mouse antihuman 565295, BD Biosciences) and CD206 FITC (anti-human, 5253911). Controls were incubated in Dako diluent without the primary antibody.

\subsubsection{Cytokine analysis}

Cell supernatants were collected 1,3 and 7 days after hTDCs treatment with IL- $1 \beta$ and PEMF stimulation and stored at $-80{ }^{\circ} \mathrm{C}$ until analysis. The supernatants were tested using commercially available enzyme immunoassay kit for IL-6 (Human IL-6 Standard ABTS ELISA Development Kit, 900-K16, Peprotech, Rocky Hill, NJ, USA) and for TNF $\alpha$ (Human TNF $\alpha$ ELISA Kit, EK0525, Tebu-Bio, Fremont, CA, USA), according to the manufacturer's instructions. Data was normalized to control conditions: hTDCs without IL- $1 \beta$ treatment.

\subsection{RNA isolation and gene expression analysis}

Total RNA was extracted using TRI reagent $(\mathrm{R})$ RNA Isolation Reagent (T9424, Sigma) following the manufacturer's instructions. RNA was quantified using a Nanodrop $\mathbb{R}$ ND-1000 spectrophotometer (Wilmington, DE, USA) at 260/280 nm. The firststrand complementary DNA was synthesized from $1 \mu \mathrm{g}$ of RNA of each sample (qScript ${ }^{\mathrm{TM}}$ cDNA Synthesis Kit, Quanta Biosciences, Gaithersburg, MD, USA) in a $20 \mu$ L reaction using a Mastercycler $\mathbb{R}$ ep realplex gradient $S$ machine (Eppendorf, Hamburg, Germany).

The quantification of the transcripts was carried out by quantitative polymerase chain reaction (qPCR) using the PerfeCTA SYBR Green FastMix kit (Quanta Biosciences, Gaithersburg, MD, USA) following the manufacturer's protocol, in a Real-Time Mastercycler ep realplex thermocycler (Eppendorf, Hamburg, Germany). The primers were designed with Primer 3 software (Supplementary Figure 1) and synthesized by MWG Biotech. The $2^{-\Delta \Delta C t}$ method was used to evaluate the relative expression level for each target gene [32].

The transcript expression of target genes was analyzed and normalized to the expression of endogenous housekeeping gene GAPDH (glyceraldehyde-3-phosphate dehydrogenase) and then to the samples collected at day $0(n=3)$.

\subsection{Statistical analysis}

Results are expressed as mean \pm standard error of the mean (SEM). The statistical analysis was performed using GraphPad Prism6 software. Data was obtained from 3-independent experiments $(n=3)$ analyzed in triplicate, and evaluated by Two-way ANOVA followed by Bonferroni post-hoc test for multiple comparison tests. A difference was considered significant with a confidence interval of $95 \%$ for different degrees of confidence, $p<0.05, p<0.01$, $p<0.001$ and $p<0.0001$.

\section{Results}

In this work, we explored the synergism of magnetic actuation and magSPCL membranes in IL- $1 \beta$ treated hTDCs to be further explored as tools to promote tendon healing and regeneration.

\subsection{Cytocompatibility and morphological characterization}

The metabolic activity of IL- $1 \beta$ treated hTDCs laden on magSPCL membranes was assessed by MTS assay (Fig. 1Ai). Metabolic activity increases in IL- $\beta$ primed cells stimulated with PEMF on day 1 and day 3 in comparison to control conditions (absence of IL- $1 \beta$ and PEMF stimulation, $p<0.01$ ). Furthermore, an increment in IL- $1 \beta$ primed cells was observed upon PEMF stimulation after 1 and 7 days in culture $(p<0.05)$. In terms of DNA content (Fig. 1Aii), IL-1 $\beta$ primed cells stimulated with PEMF showed the highest dsDNA concentration among all conditions studied $(p<0.05)$ and for all time points studied. However, the increment on the cell content with time was not statistically relevant suggesting that IL- $1 \beta$ primed cells become metabolic more active but not more proliferative.

The morphology of hTDCs was analysed by SEM (Fig. 1Aiii), showing a homogeneous distribution of hTDCs with an elongated shape on the surface of magSPCL membranes (day 1). The density of hTDCs increases with time in culture, and by day 7 several layers of hTDCs are observed, covering the magSPCL membranes. As expected, the PEMF does not negatively affect the metabolic activity neither the cell distribution on magSPCL membranes.

\subsection{Extracellular matrix and cytoskeletal organization}

The extracellular matrix (ECM) and the proteins actin and vinculin were evaluated in IL- $\beta$ treated hTDCs on magSPCL membranes (Fig. 1Bii). Independently of the time in culture, the application of PEMF causes a decrease in the MMP-1, -2, -3 expression $(p<0.0001, p<0.01, p<0.05)$ (Fig. 1Bi). Nevertheless, PEMF actuation increases TIMP-1 expression in stimulated cells $(p<0.0001)$. The higher expression values of TIMP-1 in comparison to the ones of the MMPs analysed, suggests a potential effect of PEMF actuation in the ECM remodeling process.

Staining of actin filaments ( $\alpha$-actin) and focal adhesions (vinculin) were also investigated (Fig. 1Bii) to assess the contribution of PEMF and PEMF actuated membranes in cell mechano-sensing and cell adhesion processes. Vinculin immunodetection is observed in all conditions and in both static (non-PEMF) and PEMF conditions without significant variations. However, the expression of $\alpha$ actin tends to decrease in IL- $1 \beta$ treated hTDCs under PEMF condition in comparison to untreated hTDCs after 7 days in culture.

\subsection{Expression of tendon related markers in cell laden magSPCL membranes}

Tendon related proteins were analysed on IL- $1 \beta$ treated hTDCs laden in magSPCL membranes, namely scleraxis (SCX), tenomodulin (TNMD), and collagen type I (COL1), (Fig. 2A, B, C) to assess a possible influence of pro-inflammatory factors on the maintenance of the tenogenic phenotype of hTDCs under magnetic stimulation. The expression of SCX, an early tendon marker, was increased under PEMF stimulation compared to non-stimulated (static) cells, while for the late tendon markers, TNMD and COL1, only TNMD followed this trend. Additionally, the expression of tenogenic transcription factors; SCX and Mohawk $(M K X)$ as well as of ECM proteins; collagen type I and type III (COL1A1, COL3A1), decorin 

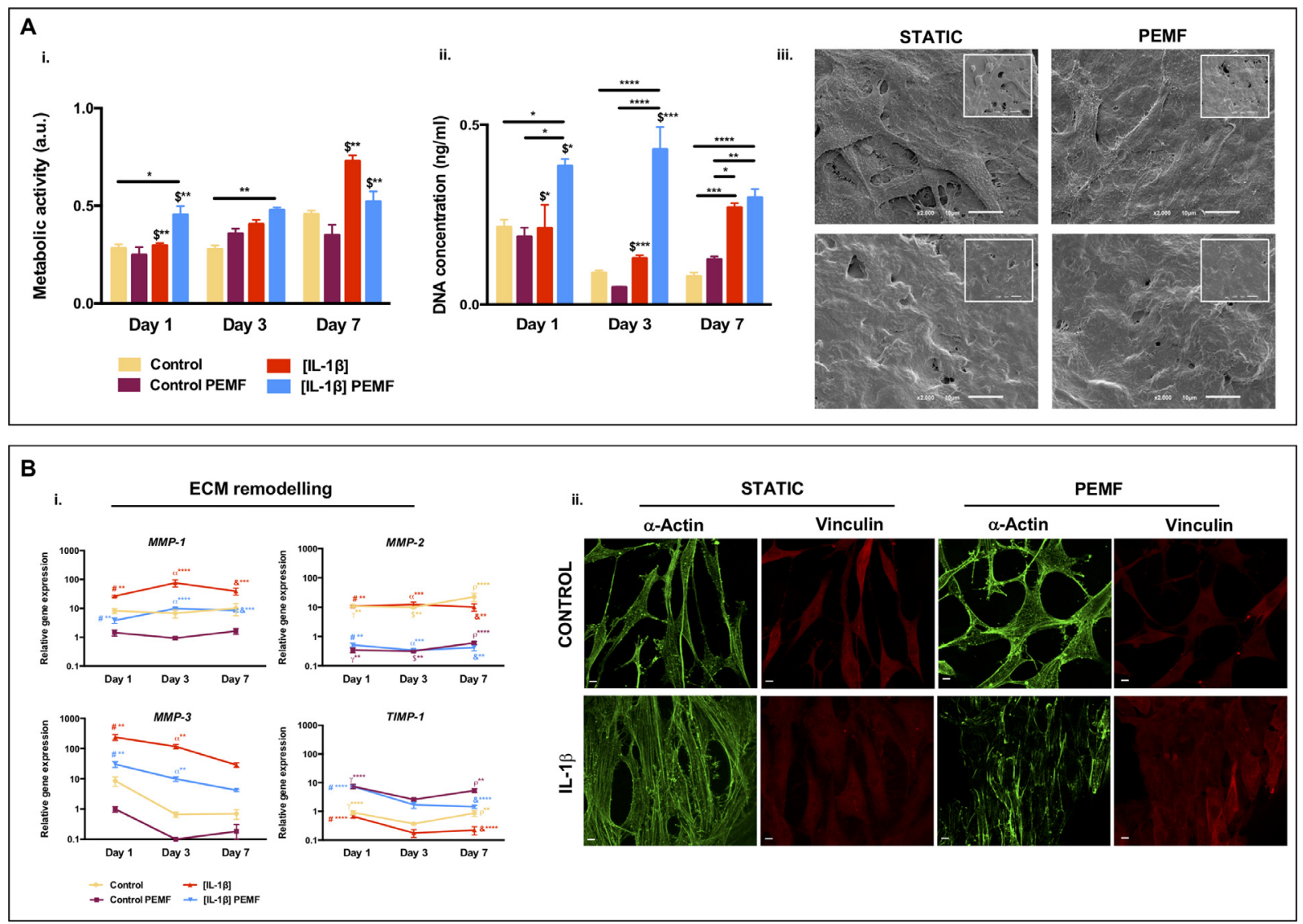

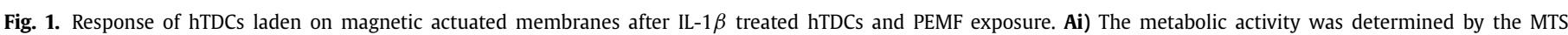

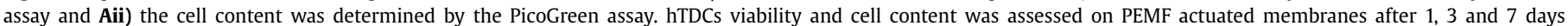

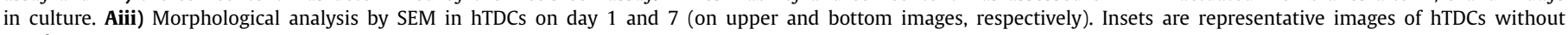

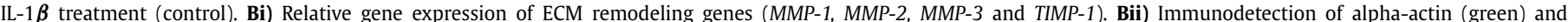

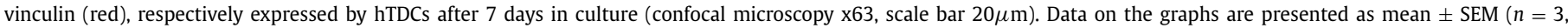

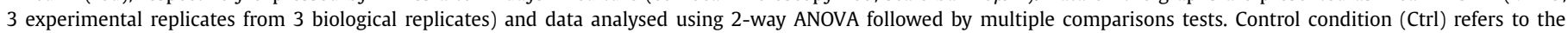

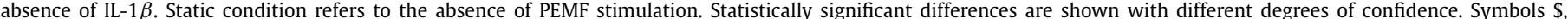

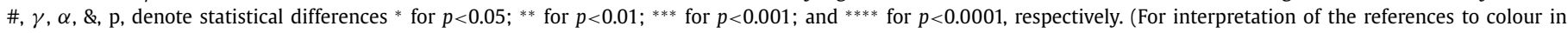
this figure legend, the reader is referred to the web version of this article.).

$(D C N)$, and tenascin $C(T N C)$ were also investigated (Fig. 2D). Overall, the genetic expression of MKX $(p<0.001), D C N(p<0.01), T N C$ $(p<0.0001)$ and collagens $(p<0.0001$ for COL1A1 and $p<0.0001$ for COL3A1, respectively) increased under PEMF application, in comparison to non-stimulated cell laden magSPCL membranes, independently of the IL- $1 \beta$ treatment. These results indicate that PEMF actuated membranes influence the gene and protein expression of tendon related-markers supporting the tenogenic phenotype in IL$1 \beta$ treated hTDCs.

\subsection{The impact of PEMF in NF-KB signaling}

The potential effect of magnetic responsive magSPCL membranes on inflammatory signaling mechanisms of IL- $\beta$ was investigated by analysing the gene expression of IL-1 $\beta$ receptor (IL1R1) and $N F-k B$ (Fig. $3 \mathrm{~A}, \mathrm{~B}$ ). In the canonical NF-kB signaling pathway IL-1 $\beta$ activates the IL-1R1 receptor, leading to an activation of IKK $\beta$ complex and this results in a translocation of NF-kB to the nucleus and further activation of target gene transcription of pro-inflammatory factors (TNF $\alpha$, IL-1 $\beta$, IL-6) (Fig. 3Ai). The expression of IL1R1 decreased on IL- $\beta$ treated cells when PEMF is applied (Fig. 3Aii). As expected, IL- $1 \beta$ treatment increased the expression of $N F-k B(p<0.01$ in comparison to non-treated hTDCs) (Fig. 3Bii). The effect of the IL- $1 \beta$ treatment seems to be antago- nized by PEMF actuation which decreases the expression of $N F-\boldsymbol{k} B$, being more evident after 3 days of culture $(p<0.0001)$.

Additionally, IL- $1 \beta$ increased the phosphorylation of NF-kB showed by a nuclear immunodetection, especially in static (nonPEMF) conditions at day 3 (Fig. 3Bi). However, when IL- $1 \beta$ treated hTDCs were stimulated with PEMF (day 3 ), the NF-kB seems to be more concentrated in the cytoplasm (Fig. 3Bii).

The activation of NF-kB signaling results in the production and release of pro-inflammatory molecules such as TNF $\alpha$, IL-6, IL-8 and COX-2. The gene expression of these cytokines (Fig. 4A) tends to decrease on PEMF stimulated constructs with or without IL- $1 \beta$. The release of IL- 6 and TNF $\alpha$ was diminished following this tendency, especially after IL- $1 \beta$ treatment $(p<0.0001$ for both IL-6 and TNF $\alpha$, in comparison to control condition) (Fig. 4C). Interestingly, IL-6 release was significantly diminished at day 7, in comparison to day 1 and $3(p<0.05)$, while TNF $\alpha$ release was maintained from day 3 to day 7 ( $p>0.05)$. Conversely, the expression of anti-inflammatory cytokines (IL-4, IL-10) tends to increase in hTDCs laden on PEMF actuated magSPCL membranes $(p<0.001$ for IL- 4 and $p<0.0001$ for IL-10, in comparison to control condition) (Fig. 4B).

Thus, PEMF seems to inhibit the expression of NF-kB in IL-1 $\beta$ treated cells, influencing the cytokine profile of hTDCs laden in magSPCL membranes. 
A

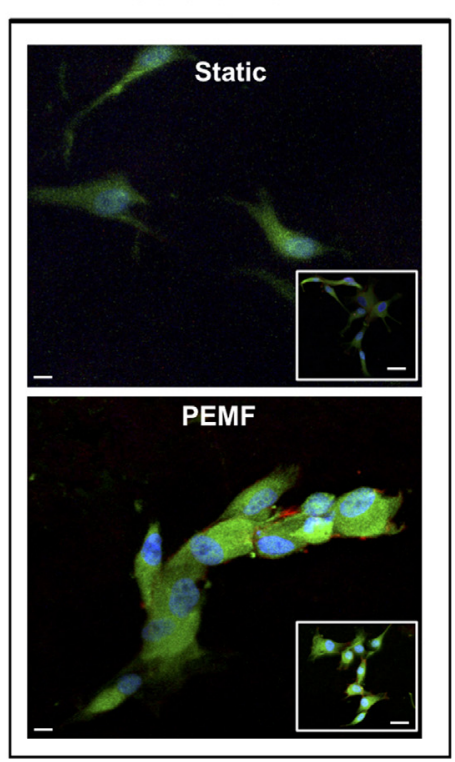

B

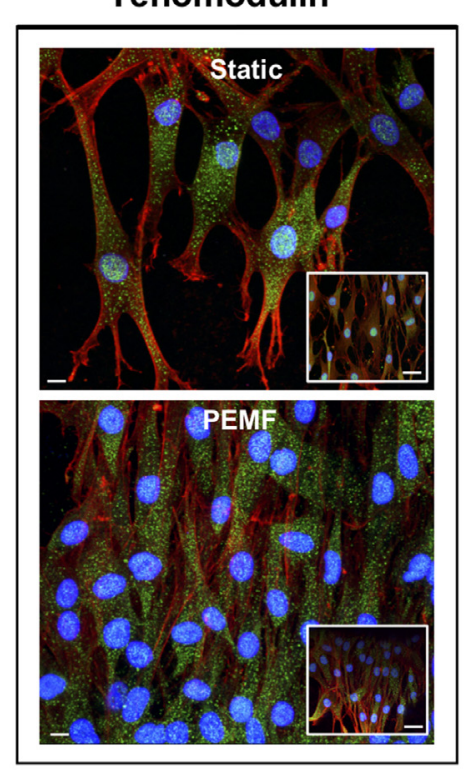

C

\section{Collagen 1}

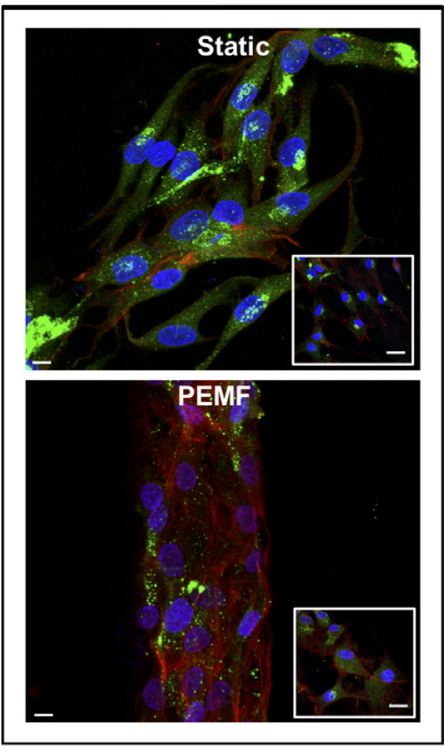

D

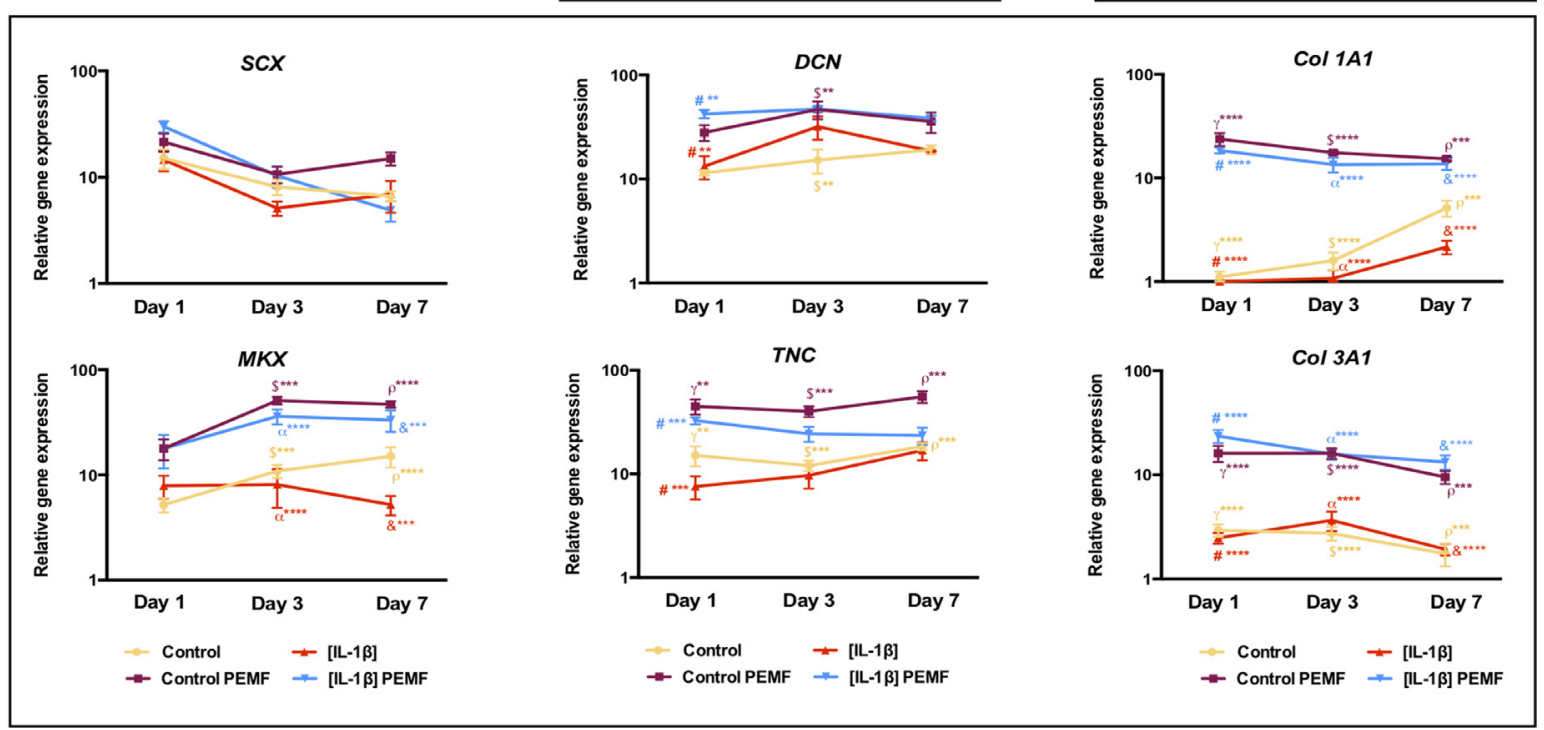

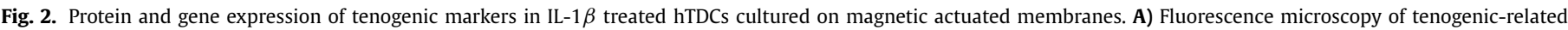

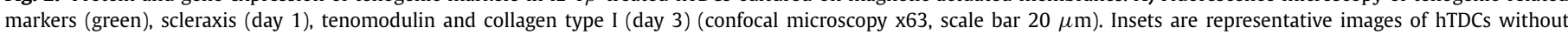

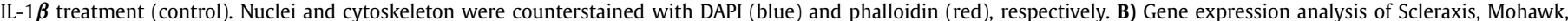

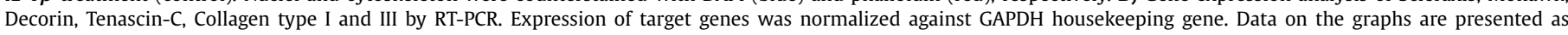

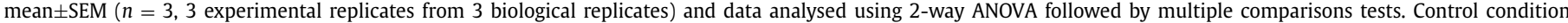

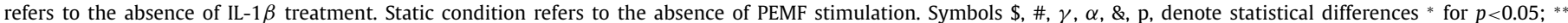

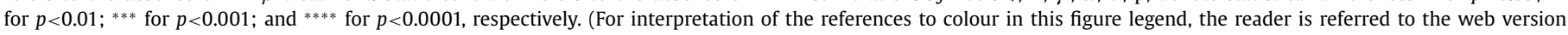
of this article.).

\subsection{Immunomodulatory potential of PEMF actuated magSPCL membranes}

The immunomodulatory action of PEMF combined with magSPCL membranes was assessed in human macrophages (Fig. 5).

Macrophages under PEMF show a more elongated shape on the surface of magSPCL membranes (Fig. $5 \mathrm{~A}$ ), which is a feature associated to a pro-healing phenotype. Further, macrophages laden on PEMF actuated magSPCL membranes were CD45+ and CD14+. Cells also expressed CD169+, a cell adhesion molecule associated to M2-type and CD68+, a M1 marker, in static conditions. Interestingly, CD16 ${ }^{++}$and $\mathrm{CD}^{206^{+}}$(M2-type) were only detected in macrophages cultured on PEMF actuated magSPCL membranes.

Altogether, results show that hTDCs and macrophages respond to the combination of PEMF stimulus and magSPCL membranes.

\section{Discussion}

The increasing attention given to magnetic actuation and magnetic responsive biomaterials has gathered promising outcomes for improving repair and regenerative mechanisms, challenges to overcome in the TERM field and in current tendon treatment regimens. Having this in mind, the combination of both could accomplish more favorable biological responses and modulate symptoms associated to impair healing.

In this work, we selected a set of PEMF parameters that antagonize pro-inflammatory cues expressed by IL- $1 \beta$ treated tendon cells [11] to be combined with magnetic responsive magSPCL membranes to enhance the pro-regenerative response of cell populations exposed to persistent inflammatory cues. MagSPCL blend has been studied in our group using various processing techniques 


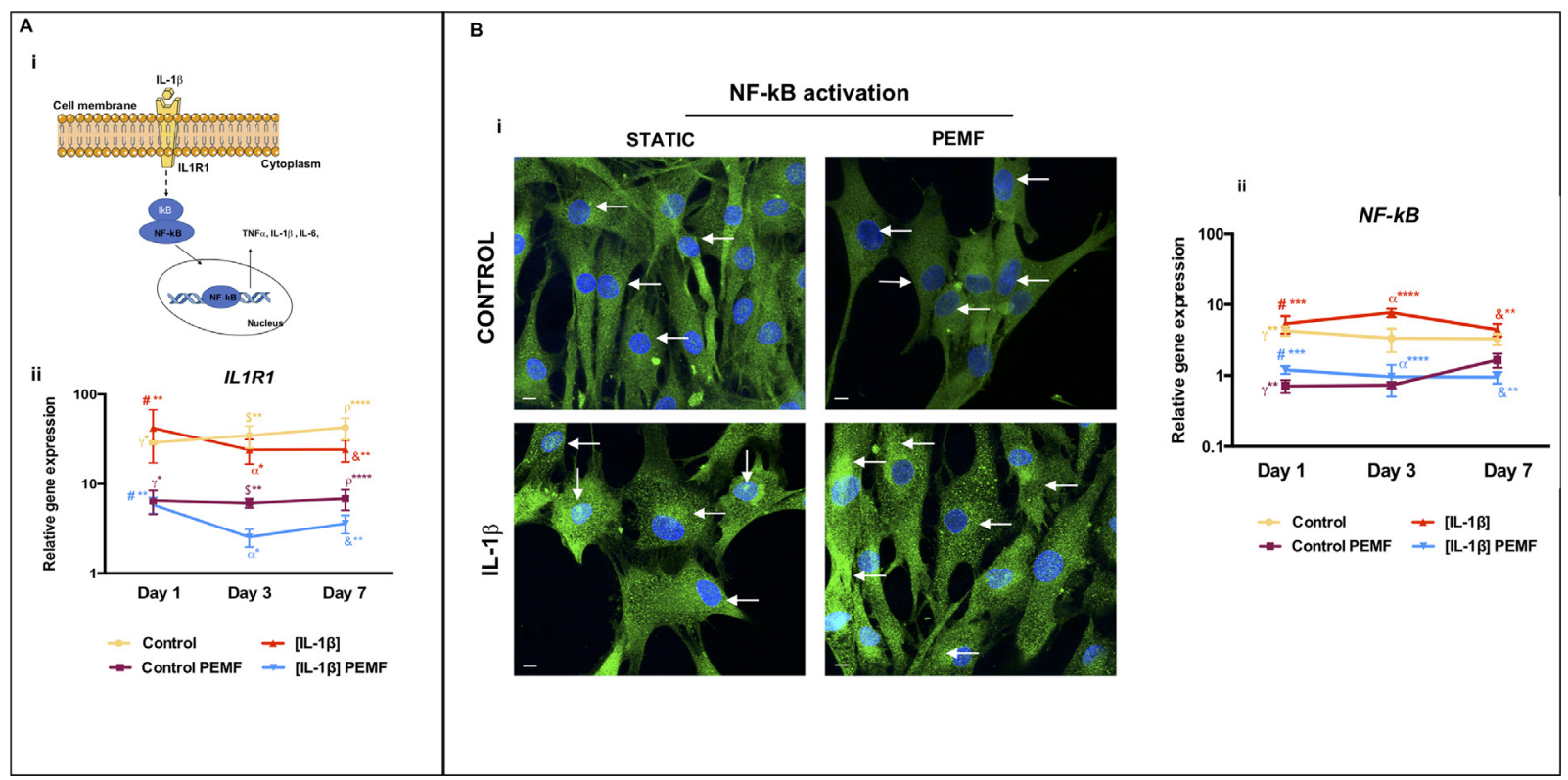

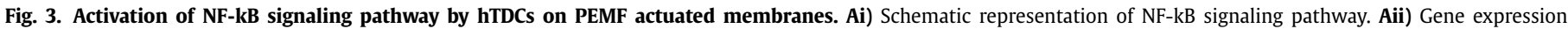

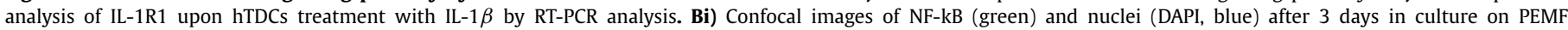

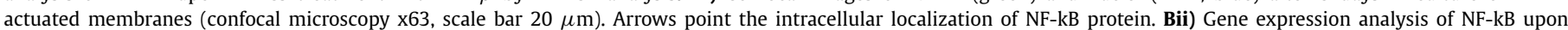

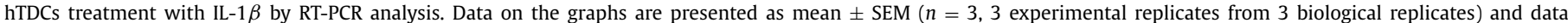

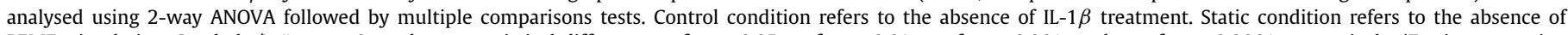

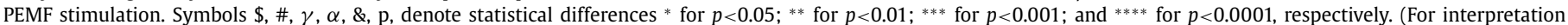
of the references to colour in this figure legend, the reader is referred to the web version of this article.).

to fabricate different shapes and architectures for magnetic based tendon tissue engineering. magSPCL matrices have shown to be biocompatible and hold immunomodulatory properties [22] in cellfree strategies.

The results obtained here showed that IL- $1 \beta$ treated tendon cells were able to colonize the magSPCL membranes, increasing their metabolic activity, especially under PEMF stimulation in the days of culture.

More importantly, the gene expression of MMPs $(-1,-2$ and -3$)$ increased in IL- $1 \beta$ treated hTDCs but, when cells were exposed to PEMF the MMPs levels decreased. The expression of MMPs, enzymes implicated in cell responses to the environmental cues, including cell proliferation, migration, differentiation and in the dynamic process of ECM turnover, with relevant implications in the inflammatory and remodeling phases of healing [33], suggest that PEMF actuation can modify hTDCs behavior, decreasing the expression of MMPs associated to collagen degradation, which may contribute to inflammatory pain and tendon lesions. As cytoskeleton changes often precede MMP modulation during tissue remodeling, actin dynamics might be linked to the expression of MMP genes [34]. In our study the detection of vinculin and $\alpha$-actin $[35,36]$ is not compromised by the PEMF stimulation. The actin expression is thus in accordance to the outcomes from MMPs, whose expression is decreased with PEMF actuation (Fig $1 \mathrm{Bi}$ and Bii).

The detection of both actin and vinculin also confirms the typical elongation of hTDCs and surface adhesion of hTDCs to magSPCL membranes, independently of IL- $1 \beta$ treatment or PEMF stimulation.

Overall, magSPCL membranes stimulated by PEMF maintain hTDCs viability, enable hTDCs adhesion and influence the turnover of the ECM antagonizing IL- $1 \beta$ effects on ECM enzymes. Afterwards, we investigated the tenogenic phenotype of hTDCs laden in PEMF responsive membranes after IL- $1 \beta$ treatment. The combination of PEMF and magSPCL membranes did not significantly change the expression of DCN, TNC and SCX during the time in culture, but stimulated an increment in $M K X$ and in collagens expression. The increment of collagen type III stimulated by PEMF may relate to its role in the regulation of collagen type I fibrillogenesis and in tissue repair. Collagen III stabilizes the repair site and contributes to the ECM remodeling process highlighting the importance of collagen type III to promote a regenerative response [37]. Thus, PEMF actuation on hTDCs/magSPCL constructs contributes to the maintenance of a tenogenic phenotype at the gene and protein level. The synergistic action of PEMF and magSPCL membranes on the expression of tendon genes blurs the pro-inflammatory stimuli of exogenous IL- $1 \beta$ supplementation to the cells.

To elucidate the mechanisms associated to the hTDCs response to PEMF and magSPCL membranes, we searched for key mediators of the IL- $1 \beta$ intracellular signaling. The NF-kB pathway regulates genes involved in multiple immune processes and prolonged activation of NF-kB may lead to inflammatory diseases [38,39]. In the canonical NF-kB signaling pathway IL- $1 \beta$ activates the IL- $1 \beta$ receptor (IL1R1) ultimately resulting in rapid and transient nuclear translocation of NF-kB, as schematically represented in Fig. 3Ai. The NF-kB translocation to the nucleus causes the activation of target gene transcription of pro-inflammatory factors as TNF $\alpha$, IL- $1 \beta$ and IL-6 [39]. Our results indicated that IL- $1 \beta$ treatment on hTDCs increases the expression of IL1R1, promotes the activation of NF$\mathbf{k B}$ after 3 days in culture and induces up-regulation of $T N F \alpha, I L-6$, $I L-8$ and $C O X-2$ and downregulation of $I L-10$ and IL-4. Interestingly, NF-kB signaling was counteracted by magSPCL membranes stimulated by PEMF. Other studies also reported significant changes in the downregulation of TNF- $\alpha$ and NF-kB in cells continuously exposed to a PEMF at $5 \mathrm{~Hz}$ [40]. Altogether, these results highlight the relevance of PEMF and magnetic responsive materials to modulate hTDCs response to environments with inflammatory signatures.

Macrophages are IL- $1 \beta$ producing cells and major orchestrators of the inflammation process. Macrophages are present in tendon niches after tendon injury and in tendinopathy conditions, and contribute with inflammatory cues, which may prolong the inflammatory phase and influence proper healing. Magnetic stimulation 

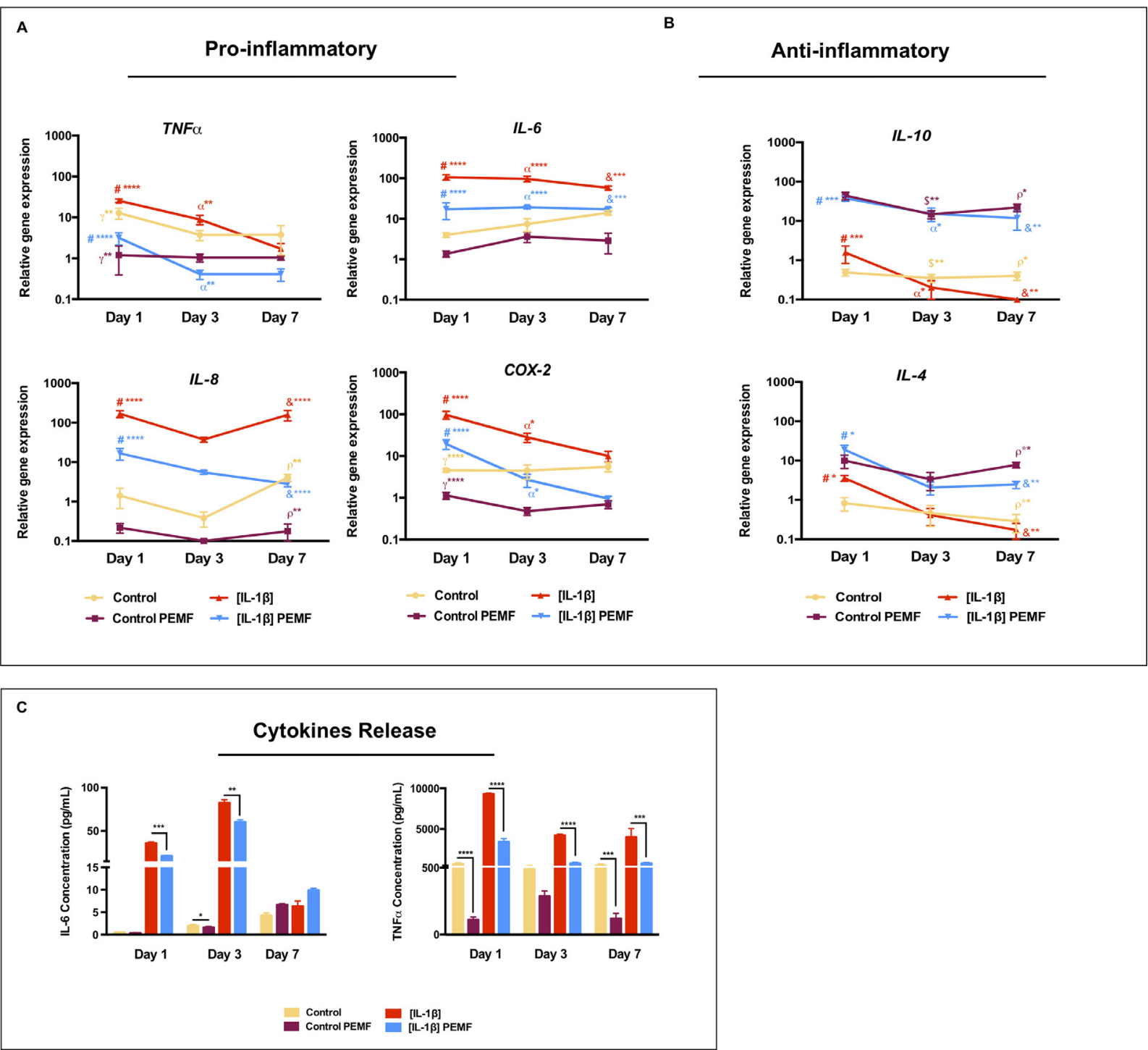

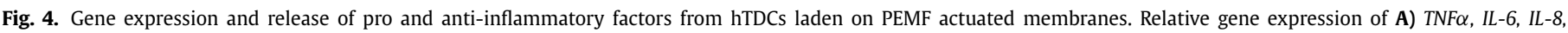

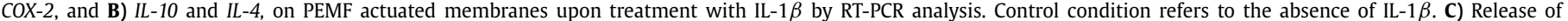

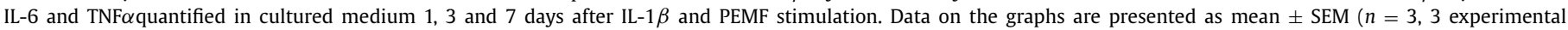

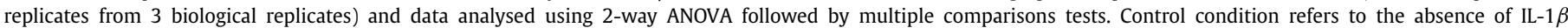

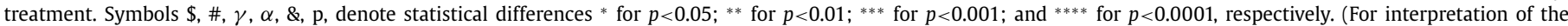
references to colour in this figure legend, the reader is referred to the web version of this article.)

seems to induce changes in macrophage morphology as these cells exhibit a more elongated shape, which has been associated to the process of macrophage polarization [41], in particular to the M2 phenotype.

Furthermore, macrophages laden in magSPCL membranes stimulated by PEMF were $\mathrm{CD}_{68}^{+}$, a M1 marker, but were also CD206 $6^{++}$ and $\mathrm{CD} 163^{++}$, which are established markers for M2 phenotype, suggesting the presence of different macrophage populations. For healing to occur, M1 macrophages should be present for the inflammatory phase and M2 macrophages are required at later stages to promote repair. Our findings suggest that PEMF responsive magSPCL membranes favor M1/M2 balance towards M2-phenotype without obliterating M1 macrophage populations. PEMF is thought to influence a number of cell-membrane processes and to activate intracellular signaling transduction. PEMF was shown to assist tenogenic phenotype [42,43], accelerate cell differentiation [44], influence the activation of cell surface receptors [45], act on voltagegated calcium channels [46] and in cell resting potential [46], which is also affected by inflammation. PEMF has also been related to inflammation kinetics, to participate in the control of tissue inflammation [47] and in the downregulation of TNF- $\alpha$ and NF-kB [40], contributing for tissue repair. The specific mechanisms in immunomodulation are outshined by the complex inflammatory cascades and intricate biological reactions mediated by different signaling pathways. Some studies also suggest a PEMF action on both transcriptional and posttranscriptional regulatory mechanisms. The involvement of PEMF in epigenetic programming [41], could explain the switch in macrophage polarization, and consequently the impact on the gene expression of mediators of the inflammatory response.

Although magSPCL membranes were approached as an in vitro model for studying cell responses under external magnetic fields and in pro-inflammatory conditions, the immunomodulatory potential of these biomaterials could be further assessed on lesions to orient cell responses, regulate pro-inflammatory events and, ultimately improve healing outcomes. Furthermore, the investigation 


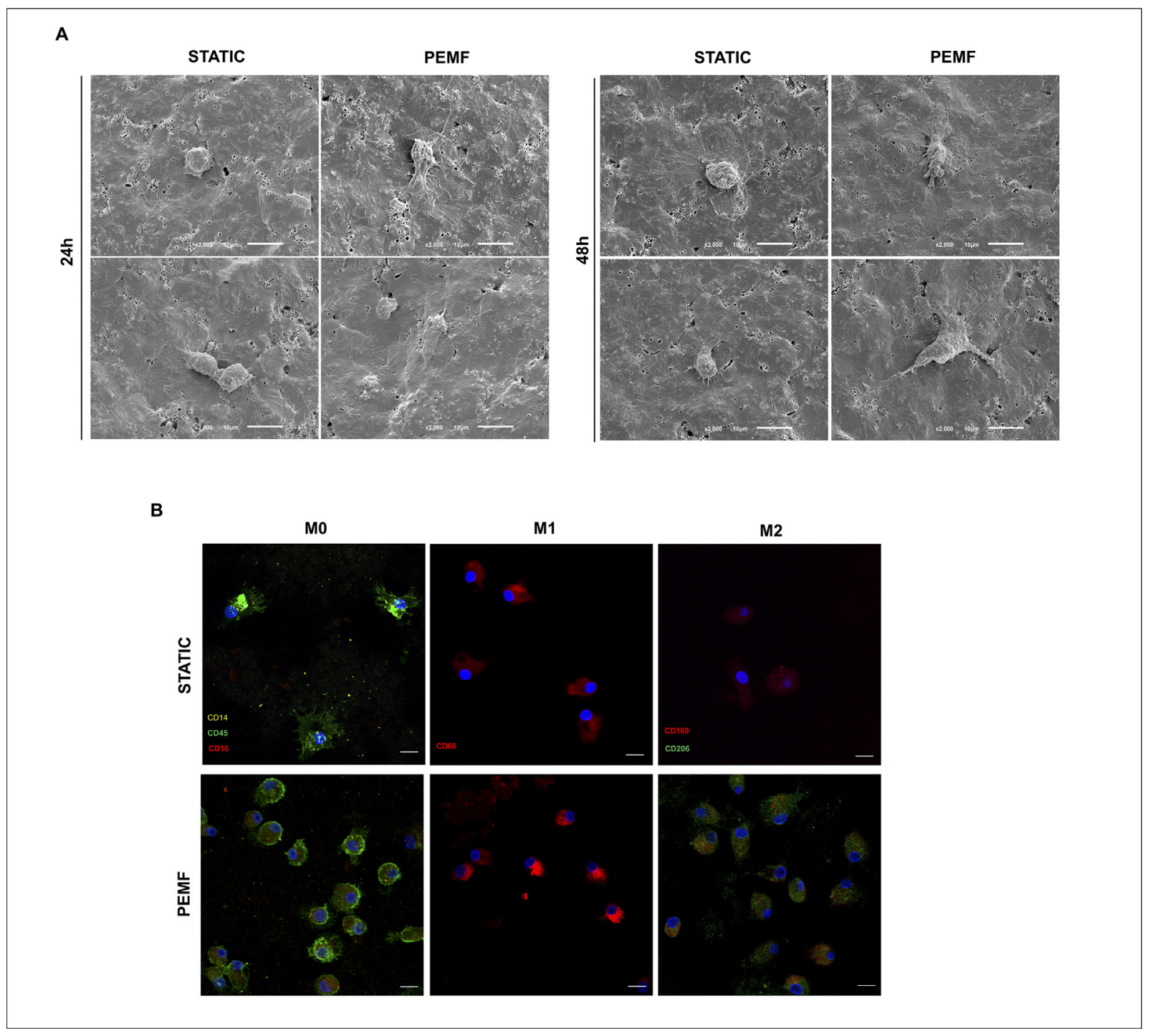

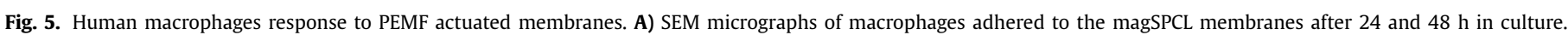

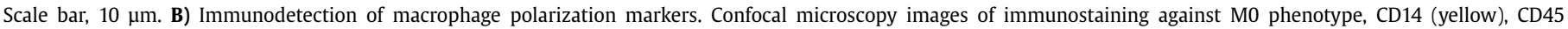

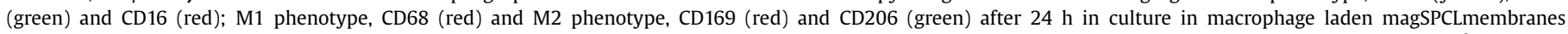

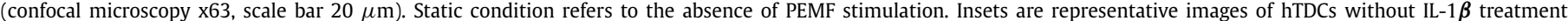
(control). (For interpretation of the references to colour in this figure legend, the reader is referred to the web version of this article.).

of cell and molecular mechanisms using commercial magnetotherapy devices used in the clinics, in which the parameters of magnetic fields are known to be safe and with therapeutic value in humans, potentially facilitates a faster translation of advanced strategies into therapeutics.

Despite the contributions of this study to the understanding of magnetic stimulation impact driving immunomodulatory cell behavior, there are some limitations to be overcome. IL- $1 \beta$ model is a widely-used model of inflammation for prospective studies but quite simplistic when compared to the pathological niches and to the intricate immune networks of living organisms. Also, the involvement of NF-kB signaling requires further investigation focusing on the precise molecules, receptors and impact on immune cells to design better strategies to address persistent inflammatory environments. In sum, PEMF and magnetic responsive materials contribute for modulating and guiding biological responses in inflammatory compromised environments associated to tendon pathologies, whose mechanisms should be deeper explored in future studies. This study highlights the complementary value of magnetic stimulus on cell laden magnetic responsive materials in guiding immunomodulatory events, controlling the perpetuation of inflammatory cues and towards resolving inflammation envisioning tendon regenerative platforms.

\section{Conclusions}

PEMF actuated magSPCL membranes have a modulatory effect on intracellular inflammatory signals and in controlling the cytokine profile of IL- $1 \beta$ treated hTDCs. The influence of magnetic forces and magnetic elements may trigger IL1R1/NF-kB signaling although it requires deeper investigation. Moreover, PEMF actuated magSPCL membranes showed an immunomodulatory effect on inflammatory cells guiding macrophages towards M2 functional programs, highlighting the potential of magnetic stimulation for tendon healing strategies.

This work contributes with new perspectives on cellular responses to pro-inflammatory environments and to the potential applicability of magnetic responsive systems as alternative therapeutic tools for the treatment of tendinopathy and for remotely stimulating tendon regeneration. 


\section{Declaration of Competing Interest}

The authors declare that they have no known competing financial interests or personal relationships that could have appeared to influence the work reported in this paper.

\section{Acknowledgments}

This research was funded by the ERC CoG grant MagTendon (No. 772817), Fundação para a Ciência e Tecnologia (FCT) Project MagTT PTDC/CTM-CTM/29930/2017, project NORTE-010145-FEDER-000021 supported by Norte Portugal Regional Operational Programme (NORTE 2020) and Achilles No. 810850. Doctoral grant PD/BD/128089/2016 of A. Vinhas was provide by FCT. The authors thank Hospital da Prelada (Porto, Portugal) for providing tendon samples.

\section{Supplementary materials}

Supplementary material associated with this article can be found, in the online version, at doi:10.1016/j.actbio.2020.09.028.

\section{References}

[1] N.L. Millar, G.A. Murrell, I.B. McInnes, Inflammatory mechanisms in tendinopathy - towards translation, Nat. Rev. Rheumatol. 13 (2) (2017) 110-122.

[2] B.J.F. Dean, S.G. Dakin, N.L. Millar, A.J. Carr, Review: emerging concepts in the pathogenesis of tendinopathy, Surgeon 15 (6) (2017) 349-354.

[3] M. Schneider, P. Angele, T.A.H. Järvinen, D. Docheva, Rescue plan for Achilles: therapeutics steering the fate and functions of stem cells in tendon wound healing, Adv. Drug Deliv. Rev. 129 (2018) 352-375.

[4] S.G. Dakin, J. Newton, F.O. Martinez, R. Hedley, S. Gwilym, N. Jones, H.A.B. Reid, S. Wood, G. Wells, L. Appleton, K. Wheway, B. Watkins, A.J. Carr, Chronic inflammation is a feature of Achilles tendinopathy and rupture, Br. J. Sports Med. (2017)

[5] S.G. Dakin, J. Dudhia, R.K. Smith, Resolving an inflammatory concept: the importance of inflammation and resolution in tendinopathy, Vet. Immunol. Immunopathol. 158 (3-4) (2014) 121-127.

[6] H.T. Xu, C.W. Lee, M.Y. Li, Y.F. Wang, P.S. Yung, O.K. Lee, The shift in macrophages polarisation after tendon injury: a systematic review, J Orthop Translat 21 (2020) 24-34

[7] M. Stolk, F. Klatte-Schulz, A. Schmock, S. Minkwitz, B. Wildemann, M. Seifert, New insights into tenocyte-immune cell interplay in an in vitro model of inflammation, Sci. Rep. 7 (1) (2017) 9801.

[8] S. Thomopoulos, W.C. Parks, D.B. Rifkin, K.A. Derwin, Mechanisms of tendon injury and repair, J. Orthop. Res. 33 (6) (2015) 832-839.

[9] L. de Girolamo, D. Stanco, E. Galliera, M. Vigano, A. Colombini, S. Setti, E. Vianello, M.M. Corsi Romanelli, V Sansone, Low frequency pulsed electromagnetic field affects proliferation, tissue-specific gene expression, and cytokines release of human tendon cells, Cell Biochem. Biophys. 66 (3) (2013) 697-708.

[10] L. de Girolamo, M. Vigano, E. Galliera, D. Stanco, S. Setti, M.G. Marazzi, G. Thiebat, M.M. Corsi Romanelli, V. Sansone, In vitro functional response of human tendon cells to different dosages of low-frequency pulsed electromagnetic field, Knee Surg. Sports Traumatol. Arthrosc. 23 (11) (2015) 3443-3453.

[11] A. Vinhas, M.T. Rodrigues, A.I. Goncalves, R.L. Reis, M.E. Gomes, Pulsed electromagnetic field modulates tendon cells response in IL-1beta conditioned environment, J. Orthop. Res. (2019).

[12] L. de Girolamo, M. Viganò, E. Galliera, D. Stanco, S. Setti, M.G. Marazzi, G. Thiebat, M.M. Corsi Romanelli, V. Sansone, In vitro functional response of human tendon cells to different dosages of low-frequency pulsed electromagnetic field, Knee Surg., Sports Traumatol., Arthrosc. 23 (11) (2014) 3443-3453.

[13] Y. Yin, P. Chen, Q. Yu, Y. Peng, Z. Zhu, J. Tian, The effects of a pulsed electromagnetic field on the proliferation and osteogenic differentiation of human adipose-derived stem cells, Med. Sci. Monit. 24 (2018) 3274-3282.

[14] M.T. Galloway, A.L. Lalley, J.T. Shearn, The role of mechanical loading in tendon development, maintenance, injury, and repair, J. Bone Joint Surg.-Am. 95 (17) (2013) 1620-1628.

[15] C.K. Kuo, R.S. Tuan, Mechanoactive tenogenic differentiation of human mesenchymal stem cells, Tissue Eng. Part A 14 (10) (2008) 1615-1627.

[16] K.H. Vining, D.J. Mooney, Mechanical forces direct stem cell behaviour in development and regeneration, Nat. Rev. Mol. Cell Biol. 18 (12) (2017) 728-742.

[17] A.I. Goncalves, M. Rotherham, H. Markides, M.T. Rodrigues, R.L. Reis, M.E. Gomes, A.J. El Haj, Triggering the activation of Activin A type II receptor in human adipose stem cells towards tenogenic commitment using mechanomagnetic stimulation, Nanomedicine 14 (4) (2018) 1149-1159.

[18] Y. Sapir-Lekhovitser, M.Y. Rotenberg, J. Jopp, G. Friedman, B. Polyak, S. Cohen, Magnetically actuated tissue engineered scaffold: insights into mechanism of physical stimulation, Nanoscale 8 (6) (2016) 3386-3399.
[19] L.J. Santos, R.L. Reis, M.E. Gomes, Harnessing magnetic-mechano actuation in regenerative medicine and tissue engineering, Trends Biotechnol. 33 (8) (2015) $471-479$.

[20] A.R. Tomas, A.I. Goncalves, E. Paz, P. Freitas, R.M.A. Domingues, M.E. Gomes, Magneto-mechanical actuation of magnetic responsive fibrous scaffolds boosts tenogenesis of human adipose stem cells, Nanoscale 11 (39) (2019) 18255-18271.

[21] S. Hao, J. Meng, Y. Zhang, J. Liu, X. Nie, F. Wu, Y. Yang, C. Wang, N. Gu, H. Xu, Macrophage phenotypic mechanomodulation of enhancing bone regeneration by superparamagnetic scaffold upon magnetization, Biomaterials 140 (2017) $16-25$.

[22] L. Santos, M. Silva, A.I. Gonçalves, T. Pesqueira, M.T. Rodrigues, M.E. Gomes, In vitro and in vivo assessment of magnetically actuated biomaterials and prospects in tendon healing, Nanomedicine 11 (9) (2016) 1107-1122.

[23] C.L. Ross, The use of electric, magnetic, and electromagnetic field for directed cell migration and adhesion in regenerative medicine, Biotechnol. Prog. 33 (1) (2017) 5-16.

[24] E.D. Silva, P.S. Babo, R. Costa-Almeida, R.M.A. Domingues, B.B. Mendes, E. Paz, P. Freitas, M.T. Rodrigues, P.L. Granja, M.E. Gomes, Multifunctional magnetic-responsive hydrogels to engineer tendon-to-bone interface, Nanomedicine (2017).

[25] A.I. Goncalves, M.T. Rodrigues, M.E. Gomes, Tissue-engineered magnetic cell sheet patches for advanced strategies in tendon regeneration, Acta Biomater 63 (2017) 110-122.

[26] C. Buhrmann, A. Mobasheri, F. Busch, C. Aldinger, R. Stahlmann, A. Montaseri, M. Shakibaei, Curcumin modulates nuclear factor kappaB (NF-kappaB)-mediated inflammation in human tenocytes in vitro: role of the phosphatidylinositol 3-kinase/Akt pathway, J. Biol. Chem. 286 (32) (2011) 28556-28566.

[27] C. Bauge, S. Leclercq, T. Conrozier, K. Boumediene, TOL19-001 reduces inflammation and MMP expression in monolayer cultures of tendon cells, BMC Complement. Altern. Med. 15 (2015) 217.

[28] J.F. Requicha, C.A. Viegas, S. Hede, I.B. Leonor, R.L. Reis, M.E. Gomes, Design and characterization of a biodegradable double-layer scaffold aimed at periodontal tissue-engineering applications, J. Tissue Eng. Regen. Med. 10 (5) (2016) 392-403.

[29] A.I. Gonçalves, R. Costa-Almeida, P. Gershovich, M.T. Rodrigues, R.L. Reis, M.E. Gomes, Cell-based approaches for tendon regeneration, in: M.E. Gomes, R.L. REIS, M.T. Rodrigues (Eds.), Tendon Regeneration. Understanding Tissue Physiology and Development to Engineer Functional Substitutes, Mica Haley, 2015, pp. 187-206.

[30] R. Costa-Almeida, L. Gasperini, J. Borges, P.S. Babo, M.T. Rodrigues, J.F. Mano, R.L. Reis, M.E. Gomes, Microengineered multicomponent hydrogel fibers: combining polyelectrolyte complexation and microfluidics, ACS Biomater. Sci. Eng. 3 (7) (2016) 1322-1331.

[31] Y. Bi, D. Ehirchiou, T.M. Kilts, C.A. Inkson, M.C. Embree, W. Sonoyama, L. Li, A.I. Leet, B.M. Seo, L. Zhang, S. Shi, M.F. Young, Identification of tendon stem/progenitor cells and the role of the extracellular matrix in their niche, Nat. Med. 13 (10) (2007) 1219-1227.

[32] K.J. Livak, T.D. Schmittgen, Analysis of relative gene expression data using real-time quantitative PCR and the 2(-Delta Delta C(T)) Method, Methods 25 (4) (2001) 402-408.

[33] J.M. Lloris-Carsi, C. Barrios, B. Prieto-Moure, J.M. Lloris-Cejalvo, D. Cejalvo-Lapena, The effect of biological sealants and adhesive treatments on matrix metalloproteinase expression during renal injury healing, PLoS ONE 12 (5) (2017) e0177665.

[34] N. Bildyug, Matrix metalloproteinases: an emerging role in regulation of actin microfilament system, Biomol. Concepts 7 (5-6) (2016) 321-329.

[35] J.L. Bays, K.A. DeMali, Vinculin in cell-cell and cell-matrix adhesions, Cell Mol. Life Sci. 74 (16) (2017) 2999-3009.

[36] A.I. Bachir, A.R. Horwitz, W.J. Nelson, J.M. Bianchini, Actin-based adhesion modules mediate cell interactions with the extracellular matrix and neighboring cells, Cold Spring Harb. Perspect. Biol. 9 (7) (2017).

[37] E.L. Miedel, B.K. Brisson, T. Hamilton, H. Gleason, G.P. Swain, L. Lopas, D. Dopkin, J.E. Perosky, K.M. Kozloff, K.D. Hankenson, S.W. Volk, Type III collagen modulates fracture callus bone formation and early remodeling, J. Orthop. Res. 33 (5) (2015) 675-684.

[38] Y. Wu, L. Chen, Y. Wang, W. Li, Y. Lin, D. Yu, L. Zhang, F. Li, Z. Pan, Overexpression of Sirtuin 6 suppresses cellular senescence and NF-kappaB mediated inflammatory responses in osteoarthritis development, Sci. Rep. 5 (2015) 17602.

[39] T. Liu, L. Zhang, D. Joo, S.C. Sun, NF-kappaB signaling in inflammation, Signal Transduct. Target Ther. 2 (2017).

[40] C.L. Ross, B.S. Harrison, Effect of pulsed electromagnetic field on inflammatory pathway markers in RAW 264.7 murine macrophages, J. Inflamm. Res. 6 (2013) 45-51.

[41] A.F. Giulia Pinton, M. Balma, L. Moro, Specific low frequency electromagnetic fields induce epigenetic and functional changes in U937 cells, Cornell University (2018) 1-14

[42] M. Liu, C. Lee, D. Laron, N. Zhang, E.I. Waldorff, J.T. Ryaby, B. Feeley, X. Liu, Role of pulsed electromagnetic fields (PEMF) on tenocytes and myoblasts-potential application for treating rotator cuff tears, J. Orthop. Res. 35 (5) (2017) 956-964.

[43] A. Marmotti, G.M. Peretti, S. Mattia, L. Mangiavini, L. de Girolamo, M. Vigano, S. Setti, D.E. Bonasia, D. Blonna, E. Bellato, G. Ferrero, F. Castoldi, Pulsed electromagnetic fields improve tenogenic commitment of umbilical cord-derived mesenchymal stem cells: a potential strategy for tendon repair-an in vitro study, Stem Cells Int. 2018 (2018) 9048237. 
[44] J. Lima, A.I. Gonçalves, M.T. Rodrigues, R.L. Reis, M.E. Gomes, The effect of magnetic stimulation on the osteogenic and chondrogenic differentiation of human stem cells derived from the adipose tissue (hASCs), J. Magn. Magn. Mater. 393 (2015) 526-536.

[45] C.L. Ross, Y. Zhou, C.E. McCall, S. Soker, T.L. Criswell, The use of pulsed electromagnetic field to modulate inflammation and improve tissue regeneration: a review, Bioelectricity 1 (4) (2019) 247-259.
[46] R.H. Funk, Coupling of pulsed electromagnetic fields (PEMF) therapy to molecular grounds of the cell, Am. J. Transl. Res. (2018).

[47] C.L. Ross, B.S. Harrison, The use of magnetic field for the reduction of inflammation: a review of the history and therapeutic results, Altern. Ther. 19 (2013) $47-54$. 